\title{
Galectin-1: the decision maker
}

The life or death of a developing thymocyte rests on the affinity of its T-cell receptor (TCR) for peptide -MHC ligands, such that a highaffinity interaction with an agonist ligand leads to death (negative selection) and an intermediateaffinity interaction with a partial agonist ligand promotes survival (positive selection). But exactly how small differences in TCR affinity are functionally translated into cell fate remains unresolved. Now, Carrie Miceli and colleagues show that galectin-1 can act as a modulator of TCR signalling in thymocytes, opposing positive selection and promoting negative selection of conventional $\mathrm{CD}^{+} \mathrm{T}$ cells.

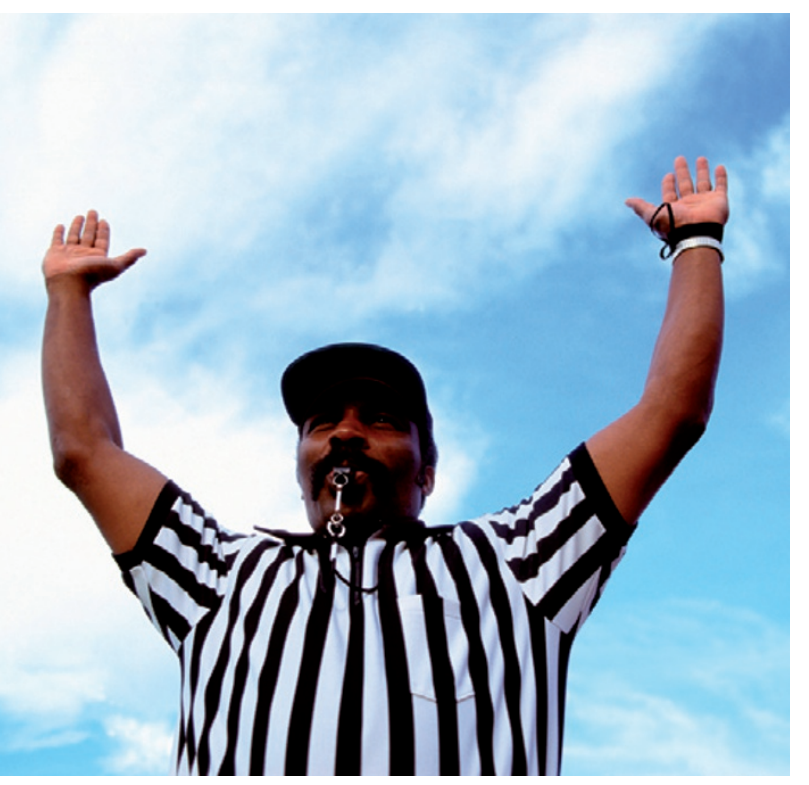

Prompted by their previous studies indicating that the galactose-binding protein galectin-1 tunes TCR signalling in peripheral T cells, Liu et al. examined the role of galectin-1 in T-cell selection using the H-Y and OT-I TCR-transgenic mouse models. In the $\mathrm{H}-\mathrm{Y}$ model, $\mathrm{H}$-Y-specific thymocytes are positively selected in female mice but negatively selected in male mice, which express the agonist ligand male antigen H-Y. Deletion of the gene encoding galectin-1 in female mice was shown to promote positive selection of $\mathrm{H}-\mathrm{Y}$ TCR ${ }^{+} \mathrm{CD}^{+}$ $\mathrm{T}$ cells, as more $\mathrm{CD} 8^{+}$thymocytes and mature $\mathrm{T}$ cells were found in these mice than in their galectin-1sufficient counterparts. Galectin-1deficient H-Y TCR-transgenic male mice also had an increased number of $\mathrm{H}-\mathrm{Y} \mathrm{TCR}^{+} \mathrm{CD}^{+} \mathrm{T}$ cells compared with control male mice, suggesting that galectin- 1 is needed for efficient negative selection. By contrast, gut-resident CD $8 \alpha \alpha^{+}$intraepithelial lymphocytes, which are known to be positively selected in the thymus by agonist ligands, were present in reduced numbers in galectin-1deficient H-Y TCR-transgenic male mice, thereby consistent with a role for galectin-1 in promoting agonist-driven selection. The role of galectin-1 in opposing positive selection was also supported by studies of OT-I TCR-transgenic mice, in which galectin-1 deficiency was shown to enhance thymocyte positive selection driven by partial agonists.
So how might galectin-1 modulate thymocyte selection thresholds? Assessment of the decay of peptide-MHC tetramer binding to TCR-transgenic thymocytes in the presence or absence of recombinant galectin-1 indicated that galectin-1 may promote agonist-driven selection by increasing binding of the TCR to agonist complexes. This hypothesis was tested by measuring the dynamics and levels of ERK (extracellularsignal-regulated kinase) activation in thymocytes exposed to CD3-specific antibody or agonist tetramer in vitro; low level sustained ERK activation is thought to result in positive selection, whereas rapid, transient ERK activation is thought to support negative selection. Consistent with the in vivo observations, the presence of galectin1 during thymocyte activation in vitro increased the intensity of the rapid and transient ERK activation that occurs on agonist-driven negative selection. By contrast, galectin-1 antagonized ERK activity in OT-I TCR ${ }^{+}$thymocytes undergoing positive selection.

Based on these observations, the authors propose that galectin-1 - possibly through the formation of glycolattice-based membrane microdomains - aids in the discrimination of TCR-directed cell-fate decisions.

Lucy Bird

ORIGINAL RESEARCH PAPER Liu, S. D. et al. Endogenous galectin-1 enforces class Irestricted TCR functional fate decisions in thymocytes. Blood 6 March 2008 (doi:10.1182/ blood-2007-09-114181) 